\title{
Innovative Progress in Simulation Languages and Applications of Simulation to Operations in Business
}

\author{
Soemon Takakuwa * \\ Graduate School of Economics and Business Administration, Nagoya University \\ Nagoya, 464-8601, Japan \\ E-mail: takakuwa@soec.nagoya-u.ac.jp \\ Rie Gaku ${ }^{\dagger}$ \\ Graduate School of Business Administration, Momoyama Gakuin University \\ Osaka, 594-1198, Japan \\ E-mail: r-gaku@andrew.ac.jp \\ Received 3 February 2014 \\ Accepted 10 April 2014
}

\begin{abstract}
System simulation has numerous applications, and plays an important role in operations management. The capability and sophistication of simulation languages have increased over the last half century. The recent progress of simulation languages from the process-oriented simulation to the object-oriented simulation is described. Then, applications of simulation to operations are introduced, presenting real examples. It is stressed that information technology is extremely important to enhancing the performance of simulation in terms of modeling and analysis.
\end{abstract}

Keywords: System simulation, Simulation language, Operations management.

\section{Introduction}

Simulation is a very broad term and implies methods and applications to imitate or mimic real systems, usually via computer. Simulation has applications in many fields and industries, including in modern business and in the area of operations (Heizer and Render 2014), and is a highly popular and powerful method.

Due to the introduction of the personal computer and animation after the late 1980s, simulation has been applied to the various systems in business, and also simulation is applied in many fields of industries as a very popular and powerful method.
Hence, first, the innovative progress of simulation languages is briefly reviewed over the last half century. Especially, the recent progress of simulation languages from the event- and the process-oriented simulation to the object-oriented simulation is illustrated. The transition from event- and process-oriented simulation to object-oriented simulation is described, particularly SIMAN, Arena, and Simio.

Then, some of remarkable reasons that the real power of simulation lies in studying complex models are described. Especially, applications of simulation to material handling, logistics, and supply chain are introduced, presenting real examples. The integration of simulation with spreadsheets is illustrated. In addition,

\footnotetext{
*Furo-cho, Chikusa-ku, Nagoya, Aichi 464-8601, Japan

†1-1 Manabino, Izumi, Osaka 594-1198, Japan
} 
the great contribution of information technology to enhancing simulation ability is described.

\section{Innovative Progress in Simulation Languages}

\subsection{Brief review of innovative progress in simulation languages}

The capabilities and sophistication of simulation languages have increased. The concept of how and when to use simulation has changed (Nance and Sargent, 2003). In the late 1950s and 1960s, simulation was a highly expensive and specialized tool that was generally used only by large corporations requiring substantial capital investments. The use of simulation began during the 1970s and early 1980s because computers were becoming faster and cheaper. During the late 1980s, simulation began to establish its real roots in business. A large part of this establishment resulted from the introduction of the personal computer and animation. Simulation truly began to mature during the 1990s. Better animation, greater ease of use, faster computers, easy integration with other packages, and the emergence of simulators all helped simulation to become a standard tool in many companies. Simulation software has taken advantage of advances in technology enabling quicker model runs, higher-fidelity animations, and comprehensive data analysis. (Kelton, Sadowski, and Swets 2010).

\subsection{Event-, process-, and object-oriented simulation}

The most basic form of simulation uses event orientation, which is simple enough in principle and has certain advantages but becomes very complicated for large models. Then, from the viewpoint of an entity as it works its way through the model, process-oriented simulation is used, as in SIMAN and Arena. In process orientation, a particular system is modeled by studying the entities that move through that system. The model consists of a description of the processes through which the entities move as they progress through the system. The word "entity" is a generic term to denote any person, object, or thing whose movement through the system causes changes in the state of the system. The term process denotes the sequence of operations or activities through which the entities move. Processes are static and are activated by entities.
In object-oriented simulation, however, the use of objects allows the modelers to reduce large problems to smaller, more manageable problems. Objects help to improve models' reliability, robustness, reusability, extensibility, and maintainability. As a result, overall modeling flexibility and power are dramatically improved. Simio employs an object approach to modeling, in which models are built by combining objects that represent the physical components of the systems. An object is a self-contained modeling construct that defines that construct's characteristics, data, behavior, user interface, and animation. Objects are now the most common constructs used to build models. A production-line model is built by placing objects that represent machines, conveyors, forklift trucks, and aisles (Kelton, Smith, and Sturrock 2013).

\section{Integration of Simulation with Spreadsheets}

Since approximately 1990, it became possible both to read external data and to write data from the simulation model to an external source. A semi-generative procedure of activity-based costing with simulation is proposed, especially for a flexible manufacturing system (Takakuwa 1997). There are two major stages in the procedure: (1) simulation and (2) activity-based costing. The scheme of this procedure is illustrated in Figure 1. Using this generative system, activity-based costing would begin automatically without inputting any data, immediately after a simulation experiment is finished and the corresponding Excel file is opened. Thus, all of the required calculations will be performed by the system.

Figure 1 illustrates the general procedure to execute a simulation experiment with the resulting external files and to generate a series of activity-based costing tables in a single Excel file. One external file is required to perform a simulation in advance, as shown in Fig. 1 (a). This file contains all data on the machining time for each specified workpart for each cutting edge at each machine tool. The animation layout for the flexible manufacturing system is shown in Fig. 1 (b).

After simulation is performed, one external file will be generated that contains the summary of machining time for each cutting edge at every machine tool. The external file is shown together with the ARENA summary report in Fig. 1 (c). Immediately after the corresponding Excel file is opened, the required data obtained by the simulation will be automatically 
inputted into the file. Four selected sheets of the Excel file are shown in Fig. 1 (d). Next, activity-based costing will be performed by filling the required cells of a series of Excel sheets sequentially and automatically. Finally, the unit manufacturing cost for each product will be obtained in the final sheet of the Excel file. Thus, activity-based costing will be performed using the simulation results.

\section{Information Technology to Enhance Simulation Ability}

Information technology such as 3D-animation display, the point of sales, electronic real-time tracking systems and global positioning systems is highly important to

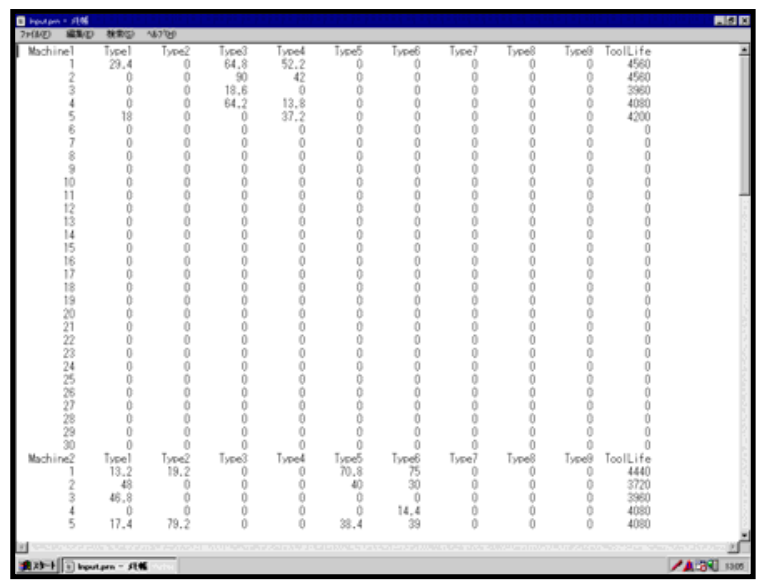

(a) External file (input).

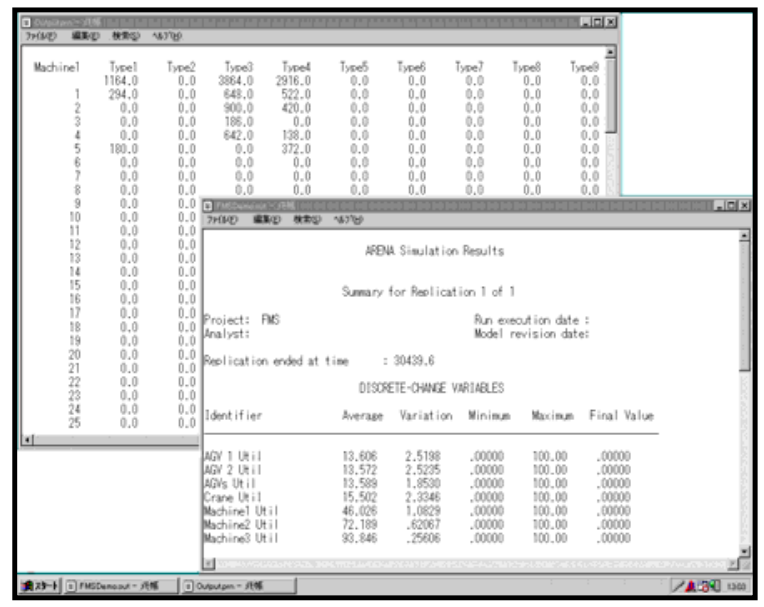

(c) Summary report and external file (output). enhancing the performance of simulation in terms of modeling. The following are real applications that use information technology.

\subsection{Electronic medical records in a general hospital}

A university hospital has prepared a plan for new hospital wards (Takakuwa and Katagiri 2007). Hence, the data on the current hospital wards are used to evaluate the performance of the new hospital wards in the study. The outpatient arrives at reception, undergoes a test, and consults a doctor. Afterward, the patient pays expenses and goes home.

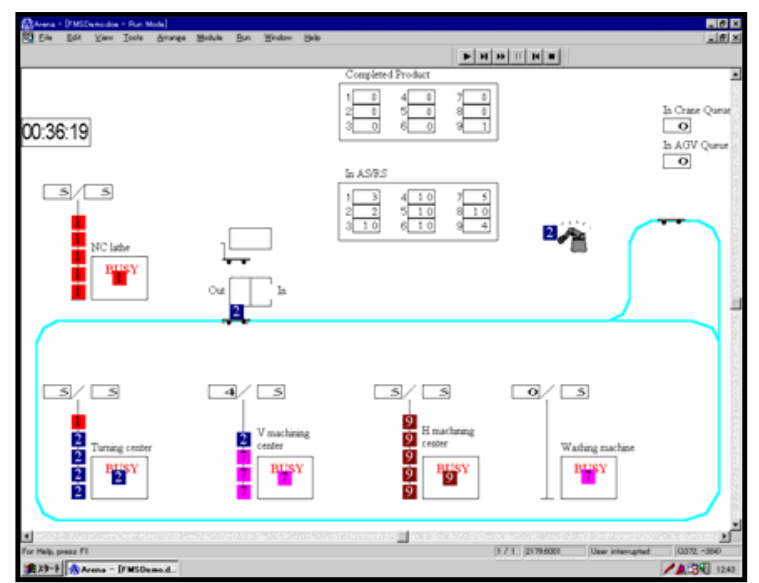

(b) Performing simulation.

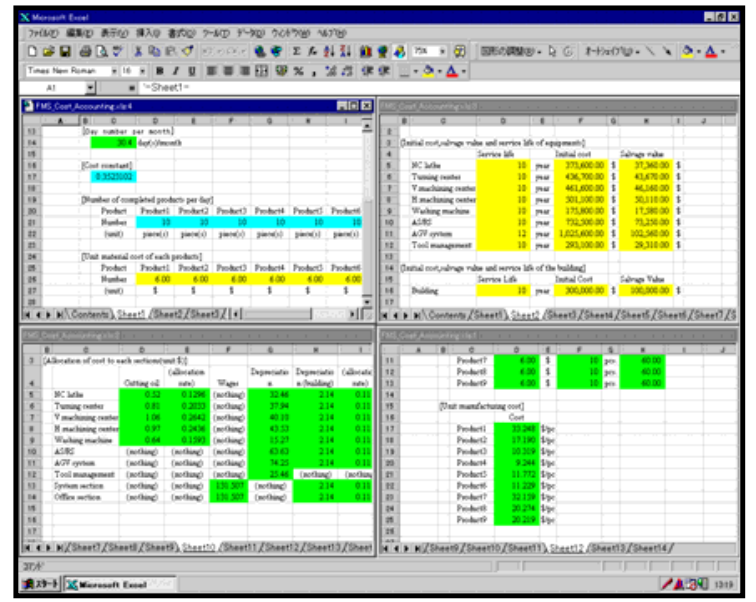

(d) Semi-generative activity-based costing system.

Fig. 1. A semi-generative procedure of activity-based costing with simulation. 
The electronic medical record is used to record the medical treatment history of the patient; in this study, it is utilized to prepare input data to perform the simulation. A layout plan and the precise work shifts of the staff are required as input data, and a time study on the testing, inspection and treatment activities should be performed as necessary. The overall flow of the data processing proposed in this study is illustrated in Fig. 2 and can be itemized as follows:

(1) Acquire a series of raw data, including reception/payment data, electronic medical record, terminal-unit data, and test/inspection data.

(2) Process the series of raw data.

(3) Prepare input data for simulation using the data generator.

(4) Perform simulation.

(5) Obtain simulation output.

An animation of the outpatient hospital ward is shown in Fig. 3.

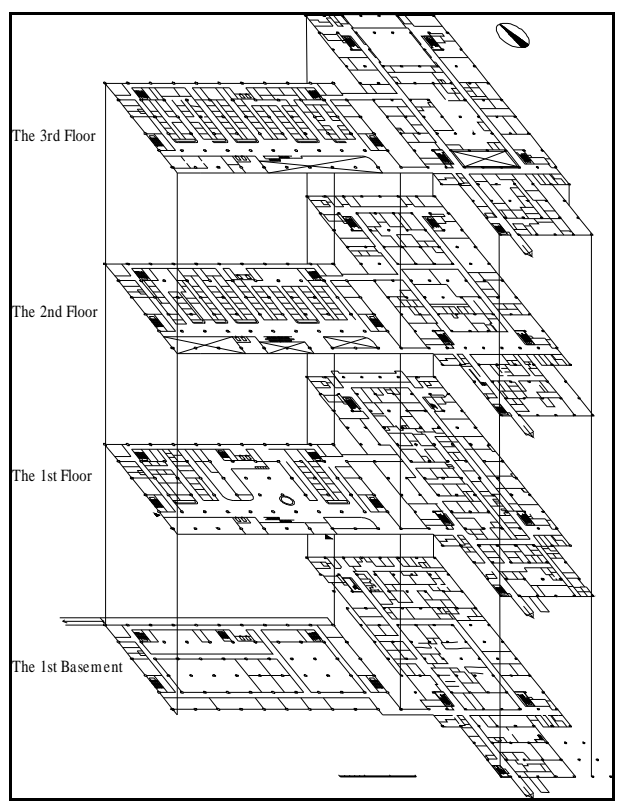

Fig. 3. Animation of outpatients hospital ward.

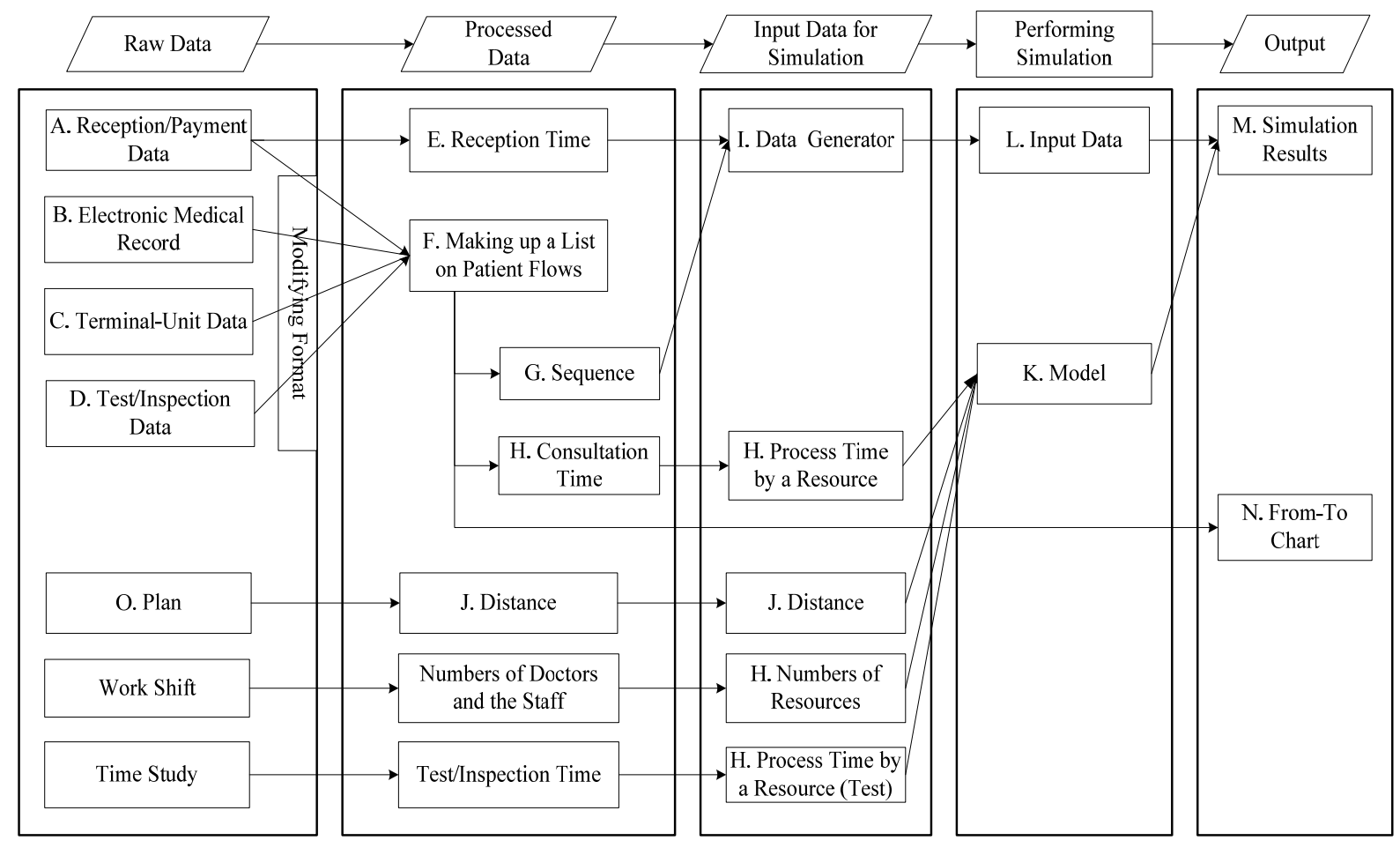

Fig. 2. Overall flow of data processing. 


\subsection{POS data at a retail store}

POS data are accumulated each day and added to customer sales information, and a procedure utilizing POS data has been proposed (Miwa and Takakuwa 2008). The proposed procedure utilizes the POS data, including the estimated arrival time and routing inside the store, as input data for the simulation model. Next, simulation is executed. Finally, the output obtained by performing the simulation is analyzed. The POS system of a retail store consists of the POS registers and the store controller. The POS register is installed at the cash desk in a store, and the store computer is placed in the room of the head of a store. When a customer purchases goods, the store clerk scans the bar code of the goods with the scanner. The scanned data are collected automatically at the new POS register. POS was newly introduced to stores in 2006, and POS data on sales transactions stored in a store computer and collected through the LAN are included as POS data. The data are processed at opening and closing every day. Next, the data are collected at the data processing center via broadband, and the collected and transmitted data are analyzed. After the records have been fully renewed, the information from the POS data is refreshed at the local office and can be observed until the following day. Fig. 4 shows a simulation of a retail store.

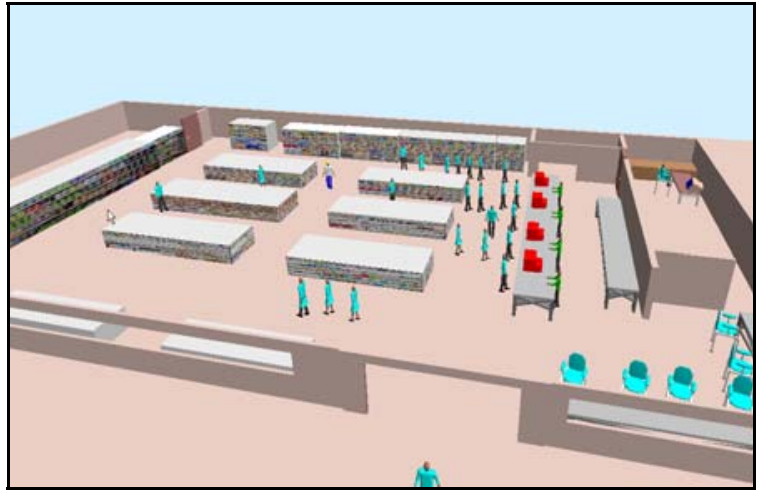

Fig. 4. Animation of a retail store.

\subsection{Electronic real-time tracking data at a container terminal}

Nagoya United Terminal System (NUTS) is a computer-aided information system used for vessel, cargo-handling equipment and yard storage planning. Fig. 5 shows the flow of container operations and the data flows via NUTS. When a ship berths at the container terminal, a container is discharged by a quay crane and unloaded onto an inside tractor to transfer to the yard storage blocks (Liu and Takakuwa 2011). Once the container is unloaded onto the bed of the tractor, the

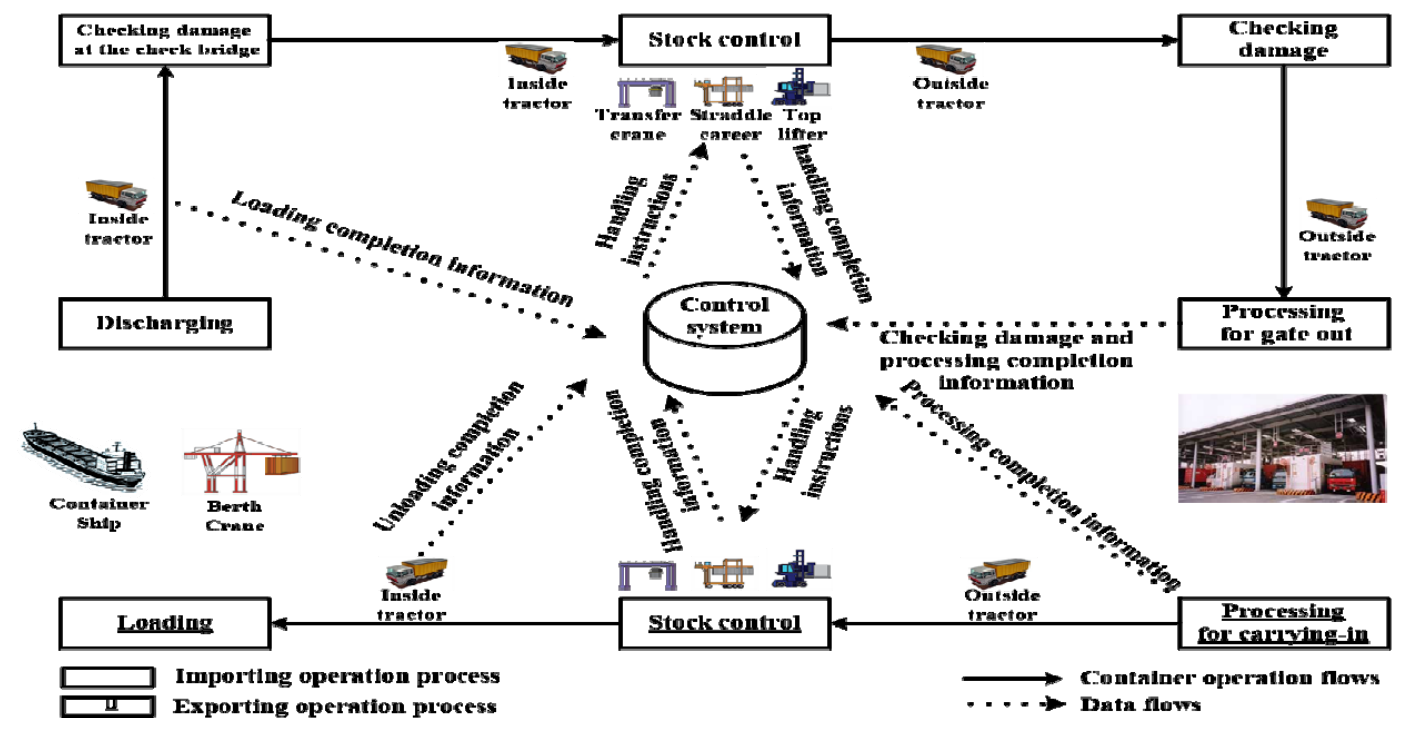

Fig. 5. Container operation flows and data flows in NUTS. 
information on loading completion will be transferred to NUTS. At nearly the same time, handling instructions for container-storage spots will be sent to cargohandling equipment at the yard, including transfer cranes, straddle careers and top lifters. It is possible to send this information to the next job in advance of the actual tractor arrival. In addition, the information is valuable for helping the cargo-handling equipment at the yard to choose the next job appropriately. Meanwhile, NUTS will record the exact time when instructions are sent out as well as when information is accepted.

In the importing process, an inbound container is discharged from an inbound vessel by a quay crane and is unloaded onto an inside tractor that transfers material to the yard storage blocks. In the exporting process, an outbound container is loaded onto a tractor by cargohandling equipment at the yard, such as transfer cranes, straddle careers, and top lifters, and then transferred to an outbound vessel by quay crane. In addition to the fundamental operation processed, there are five indispensable operational activities:

(1) Gate-in process from gate to storage yard

(2) Gate-out process from storage yard to gates

(3) Handling by transfer cranes within the same bay in a block

(4) Handling by transfer cranes between the different bays in a block

(5) Handling between the different blocks by transfer cranes and a tractor.

An animation of a container terminal is shown in Fig. 6.

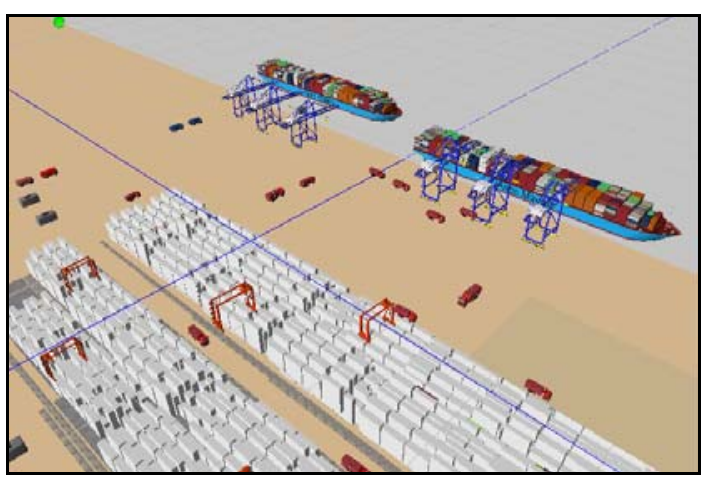

Fig. 6. Animation of a container terminal.

\subsection{GPS technology at a mining site}

Simulation involves designing a model of a real system and then conducting experiments with the model for the purpose of understanding the behavior of the system and/or of evaluating various strategies for the operation of the system (Tan, Chinbat, Miwa, and Takakuwa. 2012).

A company has introduced a mining transportation control system that utilizes GPS technology. GPS tracking data and other associated information are used to update the simulation at 1-minute intervals; important parameters, such as the truck location, the fuel level in the tank, and the load weight, are shown on the open pit map. At the designated mining site, blasting is performed at one-week intervals (on Fridays). Hence, the simulation models are constructed from the operating data collected over the course of one week. Fig. 7 shows the sample historical transportation data used in this study; these data were extracted from the database of the information system and input into the

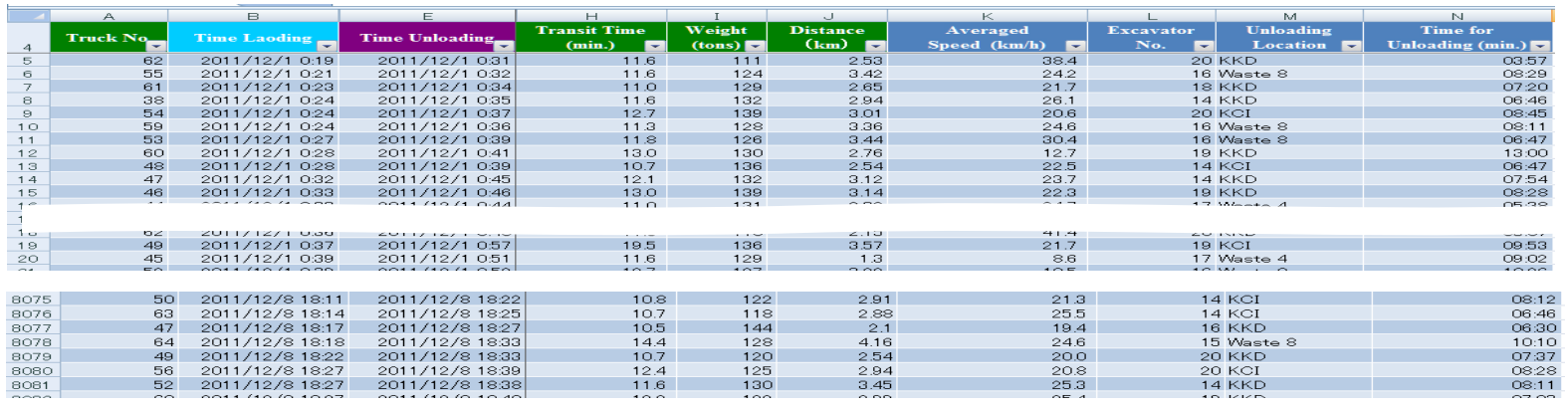

Fig. 7. The selected tracking transportation data extracted from the database of the information system. 
Simulation model as part of the simulation parameters. The simulation of an open pit mine and the associated refinery plant is shown in Fig. 8.

\subsection{Logistics information system (LIS) of the cross-docking center}

A systematic and flexible procedure is proposed and validated on a material handling operation at an actual retail-cross-docking center (Liu and Takakuwa 2009,Liu and Takakuwa 2010). The procedure uses dynamic logistics operation information, such as inbound schedules of suppliers, demand data of retail-chain stores, and individual operator schedules. The procedure is applied to an actual cross-docking center to confirm its effectiveness. Table 1 shows the order data from customers (namely from retail stores) obtained from the logistics information system (LIS) of the cross-docking center. A simulation-based approach that provides critical

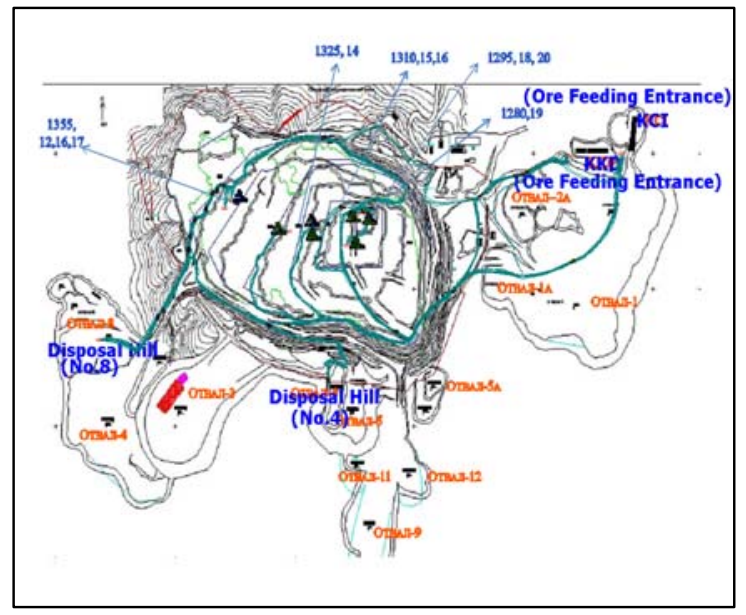

Fig. 8. Simulation of an open pit mine and the associated refinery plant. support that helps logistics managers make efficient management decisions. Animation of the cross-docking center is shown in Fig. 9.

\section{Conclusions}

(1) The recent progress of simulation languages has been quickly reviewed. Certain notable recent breakthroughs are stressed particularly from the application standpoint, introducing real examples.

(2) The difference between process-oriented simulation and object-oriented simulation, specifically between SIMAN/Arena and Simio, respectively, lies primarily in the efficiency of building simulation models, respectively.

(3) The evolution of simulation software has allowed greater integration of simulation with other packages such as spreadsheets since approximately 1990, and thus allowing the use of as large an

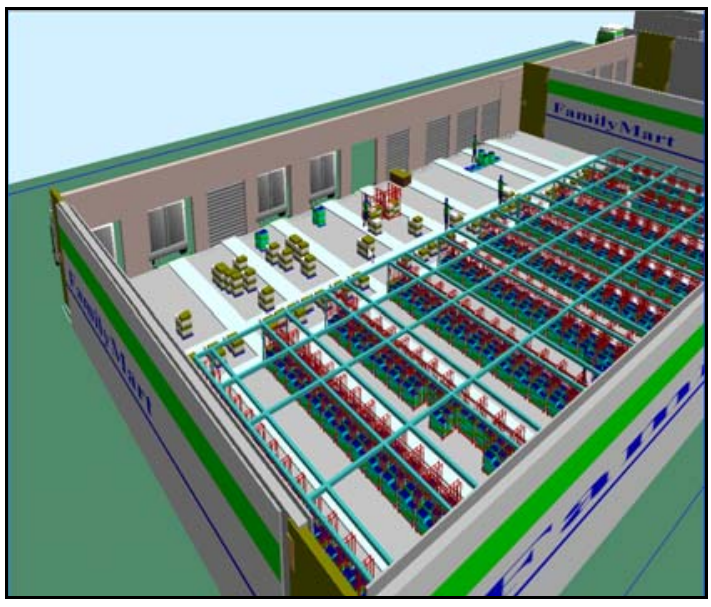

Fig. 9. Animation of the cross-docking center.

Table1: Demand Data from Stores.

\begin{tabular}{|c|c|c|c|c|c|c|c|c|c|c|c|c|}
\hline Product code & Store 1 & Store 2 & Store 3 & Store 4 & Store 5 & Store 6 & Store 7 & Store 8 & Store 9 & Store 10 & $\ldots$ & Store 236 \\
\hline 1721089 & & 4 & & & & & & & & & $\ldots$ & 3 \\
\hline 1721133 & 6 & 5 & & 3 & 3 & & & 5 & 6 & 3 & $\ldots$ & 5 \\
\hline 1748307 & 3 & & 3 & 3 & & 4 & 3 & & 3 & & $\ldots$ & 3 \\
\hline 1748314 & & & & & & 4 & 4 & 3 & & & $\ldots$ & \\
\hline 1748321 & 3 & 3 & 3 & 5 & & & 4 & & 3 & & $\ldots$ & 3 \\
\hline 1748352 & & & & & & & & & & 3 & $\ldots$ & \\
\hline 1748369 & & & & & & & & & & & $\ldots$ & \\
\hline 1748376 & 3 & & & & & & 4 & & 3 & & $\ldots$ & \\
\hline 1930061 & & & & & & & 10 & & & & $\ldots$ & 3 \\
\hline 1730067 & 9 & & & 3 & & & & & & 6 & $\ldots$ & 3 \\
\hline : & : & $:$ & $:$ & : & : & : & : & : & : & $:$ & : & : \\
\hline
\end{tabular}


amount of data has been required. This capability is extremely important in building a large-scale simulation model such as a logistics model.

(4) Applications of simulation to material handling, logistics, and supply chain are introduced, presenting real examples. It is stressed that information technology such as 3D-animation display, the point of sales, electronic real-time tracking systems and global positioning systems are extremely important to enhancing the performance of simulation in terms of modeling and analysis.

\section{Acknowledgments}

This research was supported by the Grant-in-Aid for young Scientists (B) of the Japan Society for the Promotion of Science (JSPS). The Grant Number is 23730347.

\section{References}

1. J. Heizer and B. Render, Operations management, 11th edn. (PEASON, NY, NY, 2014).

2. W W.D. Kelton, R.P. Sadowski, and N.B. Swets, Simulation with ARENA, 5th edn. (McGraw-Hill, Inc., NY, NY.,2010). / S. Takakuwa (ed.), Simulation with ARENA, 4th edn. (Japanese edition, Corona-sha, 2007).

3. W.D. Kelton, J.S. Smith, and D.T. Sturrock, Simio and Simulation: Modeling, Analysis, and Applications, 3rd edn. (Simio LLC, 2014). / S. Takakuwa (ed.), Simio and Simulation: Modeling, Analysis, and Applications, 2nd edn. (Japanese edition, Simio LLC, 2014).

4. Y. Liu and S. Takakuwa, Modeling of materials handling in a container terminal by using electronic real-time tracking data, in Proceedings of 2011 Winter Simulation Conference, pp. 1596-1604.

5. K. Miwa and S. Takakuwa, Simulation modeling and analysis for in-store merchandizing of retail stores with enhanced information technology, in Proceedings of 2008 Winter Simulation Conference, pp. 1702-1710.

6. R.E. Nance and R.G. Sargent, Perspectives on the evolution of simulation, Operations Research. 50 (2003) 161-172.

7. C.D. Pegden, Introduction to SIMAN (Systems Modeling Corporation, State College, PA, 1983). / S. Takakuwa, Introduction to SIMAN (Japanese edition, Corona-sha, 1987).

8. C.D. Pegden, R.E. Shannon and R.P. Sadowski, Introduction to Simulation Using SIMAN, 2nd edn. (McGraw-Hill, Inc., NY, NY., 1990) / S. Takakuwa, Introduction to Simulation Using SIMAN, 2nd edn. (Japanese edition, Corona-sha, 1993).

9. S. Takakuwa, The use of simulation in activity-based costing for flexible manufacturing systems, in
Proceedings of 1997 Winter Simulation Conference, pp. 793-800.

10. S. Takakuwa, and D. Katagiri, Modeling of patient flows in a large-scale outpatient hospital ward by making use of electronic medical records, in Proceedings of 2007 Winter Simulation Conference, pp. 1523-1531.

11. Y. Tan, U. Chinbat, K. Miwa and S. Takakuwa, Operations modeling and analysis of open pit copper mining using GPS tracking data, in Proceedings of 2012 Winter Simulation Conference, pp. 1309-1320.

12. Y. Liu and S. Takakuwa, Simulation-based personnel planning for materials handling at a cross-docking center under retail distribution environment, in Proceedings of 2009 Winter Simulation Conference, pp. 2414-2425.

13. Y. Liu and S. Takakuwa, Enhancing simulation as a decision-making support tool for a cross-docking center in a dynamic retail-distribution environment, in Proceedings of 2010 Winter Simulation Conference, pp. 2089-2099. 